\title{
THE CLOSURE OF DIRICHLET SPACES IN THE BLOCH SPACE
}

\author{
Petros Galanopoulos and Daniel Girela \\ Aristotle University of Thessaloniki, Department of Mathematics \\ 54124, Thessaloniki, Greece; petrosgala@math.auth.gr \\ Universidad de Málaga, Análisis Matemático \\ Campus de Teatinos, 29071 Málaga, Spain; girela@uma.es
}

\begin{abstract}
If $0<p<\infty$ and $\alpha>-1$, the space of Dirichlet type $\mathcal{D}_{\alpha}^{p}$ consists of those functions $f$ which are analytic in the unit disc $\mathbf{D}$ and have the property that $f^{\prime}$ belongs to the weighted Bergman space $A_{\alpha}^{p}$. Of special interest are the spaces $\mathcal{D}_{p-1}^{p}(0<p<\infty)$ and the analytic Besov spaces $B^{p}=\mathcal{D}_{p-2}^{p}(1<p<\infty)$. Let $\mathcal{B}$ denote the Bloch space. It is known that the closure of $B^{p}(1<p<\infty)$ in the Bloch norm is the little Bloch space $\mathcal{B}_{0}$. A description of the closure in the Bloch norm of the spaces $H^{p} \cap \mathcal{B}$ has been given recently. Such closures depend on $p$. In this paper we obtain a characterization of the closure in the Bloch norm of the spaces $\mathcal{D}_{\alpha}^{p} \cap \mathcal{B}(1 \leq p<\infty$, $\alpha>-1)$. In particular, we prove that for all $p \geq 1$ the closure of the space $\mathcal{D}_{p-1}^{p} \cap \mathcal{B}$ coincides with that of $H^{2} \cap \mathcal{B}$. Hence, contrary with what happens with Hardy spaces, these closures are independent of $p$. We apply these results to study the membership of Blaschke products in the closure in the Bloch norm of the spaces $\mathcal{D}_{\alpha}^{p} \cap \mathcal{B}$.
\end{abstract}

\section{Introduction and main results}

Let $\mathbf{D}=\{z \in \mathbf{C}:|z|<1\}$ denote the open unit disc in the complex plane $\mathbf{C}, \partial \mathbf{D}$ will be the unit circle. Also, $d A$ will denote the area measure on $\mathbf{D}$, normalized so that the area of $\mathbf{D}$ is 1 . Thus $d A(z)=\frac{1}{\pi} d x d y=\frac{1}{\pi} r d r d \theta$. The space of all analytic functions in $\mathbf{D}$ will be denoted by $\mathcal{H o l}(\mathbf{D})$. We also let $H^{p}(0<p \leq \infty)$ be the classical Hardy spaces. We refer to [9] for the notation and results regarding Hardy spaces. The space $B M O A$ consists of those functions $f \in H^{1}$ whose boundary values have bounded mean oscillation on $\partial \mathbf{D}$. The "little oh" version of $B M O A$ is the space $V M O A$. We refer to [15] for the theory of $B M O A$-functions.

For $0<p<\infty$ and $\alpha>-1$ the weighted Bergman space $A_{\alpha}^{p}$ consists of those $f \in \mathcal{H o l}(\mathbf{D})$ such that

$$
\|f\|_{A_{\alpha}^{p}} \stackrel{\text { def }}{=}\left((\alpha+1) \int_{\mathbf{D}}\left(1-|z|^{2}\right)^{\alpha}|f(z)|^{p} d A(z)\right)^{1 / p}<\infty .
$$

The unweighted Bergman space $A_{0}^{p}$ is simply denoted by $A^{p}$. We refer to $[10,19,31]$ for the notation and results about Bergman spaces. The space of Dirichlet type $\mathcal{D}_{\alpha}^{p}$ $(0<p<\infty$ and $\alpha>-1)$ consists of those $f \in \mathcal{H} o l(\mathbf{D})$ such that $f^{\prime} \in A_{\alpha}^{p}$. In other

https://doi.org/10.5186/aasfm.2019.4402

2010 Mathematics Subject Classification: Primary 30H30; Secondary 46E15.

Key words: Bloch space, Dirichlet spaces, Besov spaces, weighted Bergman spaces, closure in the Bloch norm, Blaschke product.

This research is supported by grants from "El Ministerio de Economía y Competitividad, Spain" MTM2014-52865-P; and from "La Junta de Andalucía" (FQM210). 
words, a function $f \in \mathcal{H} o l(\mathbf{D})$ belongs to $\mathcal{D}_{\alpha}^{p}$ if and only if

$$
\|f\|_{\mathcal{D}_{\alpha}^{p}} \stackrel{\text { def }}{=}|f(0)|+\left((\alpha+1) \int_{\mathbf{D}}\left(1-|z|^{2}\right)^{\alpha}\left|f^{\prime}(z)\right|^{p} d A(z)\right)^{1 / p}<\infty .
$$

If $\alpha>p-1$ then it is well known that $\mathcal{D}_{\alpha}^{p}=A_{\alpha-p}^{p}$ (see, e.g., [11, Theorem 6]). For $1<p<\infty$, the space $\mathcal{D}_{p-2}^{p}$ is the analytic Besov space $B^{p}$. The space $B^{1}$ requires a special definition: it is the space of all functions $f \in \mathcal{H o l}(\mathbf{D})$ such that $f^{\prime \prime} \in A^{1}$. Although the corresponding semi-norm is not conformally invariant, the space itself is. Another possible definition (with a conformally invariant semi-norm) is given in the fundamental article [3], where $B^{1}$ was denoted by $\mathcal{M}$. The spaces $B^{p}$, $1 \leq p<\infty$, form a nested scale of conformally invariant spaces which are contained in $V M O A$ and show up naturally in different settings (see [3], [8] and [30]). In particular, $\mathcal{D}_{0}^{2}=B^{2}$ is the classical Dirichlet space $\mathcal{D}$.

Finally, we recall that a function $f \in \mathcal{H} o l(\mathbf{D})$ is said to be a Bloch function if

$$
\|f\|_{\mathcal{B}} \stackrel{\text { def }}{=}|f(0)|+\sup _{z \in \mathbf{D}}\left(1-|z|^{2}\right)\left|f^{\prime}(z)\right|<\infty .
$$

The space of all Bloch functions will be denoted by $\mathcal{B}$. It is a non-separable Banach space with the norm $\|\cdot\|_{\mathcal{B}}$ just defined. A classical source for the theory of Bloch functions is [1]. The closure of the polynomials in the Bloch norm is the little Bloch space $\mathcal{B}_{0}$ which consists of those $f \in \mathcal{H}$ ol $(\mathbf{D})$ with the property that

$$
\lim _{|z| \rightarrow 1}\left(1-|z|^{2}\right)\left|f^{\prime}(z)\right|=0 \text {. }
$$

It is well known that

$$
H^{\infty} \subsetneq B M O A \subsetneq \cap_{0<p<\infty} H^{p}, \quad H^{\infty} \subsetneq B M O A \subsetneq \mathcal{B}, \quad V M O A \subsetneq \mathcal{B}_{0} \subsetneq \mathcal{B} .
$$

Anderson, Clunie and Pommerenke [1, p. 36] raised the question of determining the closure of $H^{\infty}$ in $\mathcal{B}$. They remarked that this closure strictly contains $\mathcal{B}_{0}$ but is not identical with $\mathcal{B}$. The problem is still open. However, Jones gave an unpublished description of the closure of $B M O A$ in $\mathcal{B}$ (see [2, Theorem 9]). Given $f \in \mathcal{B}$ and $\varepsilon>0$, we define

$$
\Omega_{\varepsilon}(f)=\left\{z \in \mathbf{D}:\left(1-|z|^{2}\right)\left|f^{\prime}(z)\right| \geq \varepsilon\right\} .
$$

Then a Bloch function $f$ is in the closure of $B M O A$ in the Bloch norm if and only if for every $\varepsilon>0$ the Borel measure $\left(1-|z|^{2}\right)^{-1} \chi_{\Omega_{\varepsilon}(f)}(z) d A(z)$ is a Carleson measure in D. As usual, for a Borel subset $E$ of $\mathbf{D}, \chi_{E}$ denotes the characteristic function of E. A proof of Jones' description is provided by Ghatage and Zheng [14].

This study has been broaden to determine the closure in the Bloch norm of other subspaces of $\mathcal{B}$. For simplicity, if $X$ is a subspace of the Bloch space we shall let $\mathcal{C}_{\mathcal{B}}(X)$ denote the closure in the Bloch norm of the space $X$.

Tjani [26] proved that if $f \in \mathcal{B}$, then $f \in \mathcal{B}_{0}$ if and only if $\int_{\Omega_{\varepsilon}(f)} \frac{d A(z)}{\left(1-|z|^{2}\right)^{2}}<\infty$ for every $\varepsilon>0$. Since all Besov spaces contain the polynomials and are contained in $\mathcal{B}_{0}$, we have

$$
\mathcal{C}_{\mathcal{B}}\left(B^{p}\right)=\mathcal{B}_{0}, \quad 1 \leq p<\infty .
$$

This was observed in [29] where the closures in the Bloch norm of other conformally invariant spaces were also described. Bao and Göğüs [5] have recently characterized the closure in the Bloch norm of the space $\mathcal{D}_{\alpha}^{2} \cap \mathcal{B}(-1<\alpha \leq 1)$. 
Monreal Galán and Nicolau [22] described the closure in the Bloch norm of $\mathcal{B} \cap H^{p}$, for $1<p<\infty$. Galanopoulos, Monreal Galán and Pau [13] have extended this result to the whole range $0<p<\infty$.

Let us fix some notation. Given a Lebesgue measurable subset $\Omega$ of $\mathbf{D}$, we let $A_{h}(\Omega)$ be the hyperbolic area of $\Omega$, that is,

$$
A_{h}(\Omega)=\int_{\Omega} \frac{d A(z)}{\left(1-|z|^{2}\right)^{2}}
$$

Also, for fixed $a>1$ and for $\xi \in \partial \mathbf{D}$, we let $\Gamma_{a}(\xi)=\{z \in \mathbf{D}:|z-\xi|<a(1-|z|)\}$ be the Stolz angle with vertex at $\xi$. Putting [22, Theorem 1] and [13, Theorem 1] together yields the following result.

Theorem A. Let $0<p<\infty$ and $a>1$. A Bloch function $f$ is in the closure in the Bloch norm of $\mathcal{B} \cap H^{p}$ if and only if for every $\varepsilon>0$ the function $F_{\varepsilon}(f)$ defined by

$$
F_{\varepsilon}(f)(\xi)=A_{h}^{1 / 2}\left(\Gamma_{a}(\xi) \cap \Omega_{\varepsilon}(f)\right), \quad \xi \in \partial \mathbf{D},
$$

belongs to $L^{p}(\partial \mathbf{D})$, that is,

$$
\int_{\partial \mathbf{D}}\left(\int_{\Gamma_{a}(\xi) \cap \Omega_{\varepsilon}(f)} \frac{d A(z)}{\left(1-|z|^{2}\right)^{2}}\right)^{p / 2}|d \xi|<\infty .
$$

It is well known that there exists a positive constant $C$ such that

$$
|f(z)| \leq C\|f\|_{\mathcal{B}} \log \frac{2}{1-|z|}, \quad(z \in \mathbf{D}), \text { for every } f \in \mathcal{B},
$$

(see [1, p. 13]). Then it follows trivially that $\mathcal{B} \subset A_{\alpha}^{p}$ whenever $0<p<\infty$ and $\alpha>-1$. So the question of characterizing $\mathcal{C}_{\mathcal{B}}\left(A_{\alpha}^{p} \cap \mathcal{B}\right)$ is trivial:

$$
\mathcal{C}_{\mathcal{B}}\left(A_{\alpha}^{p} \cap \mathcal{B}\right)=\mathcal{C}_{\mathcal{B}}(\mathcal{B})=\mathcal{B}, \quad 0<p<\infty, \quad \alpha>-1
$$

The main object of this paper is to characterize the closure in the Bloch norm of the spaces $\mathcal{D}_{\alpha}^{p} \cap \mathcal{B}$. As we mentioned above, if $p-1<\alpha$ then $\mathcal{D}_{\alpha}^{p}=A_{\alpha-p}^{p}$. Thus, using (1.2) we obtain

$$
\mathcal{C}_{\mathcal{B}}\left(\mathcal{D}_{\alpha}^{p} \cap \mathcal{B}\right)=\mathcal{B}, \quad 0<p<\infty, \quad p-1<\alpha .
$$

If $-1<\alpha \leq p-2$ then we have that $\mathcal{D}_{\alpha}^{p} \subset \mathcal{D}_{p-2}^{p}=B^{p} \subset \mathcal{B}$, and then (1.1) implies that

$$
\mathcal{C}_{\mathcal{B}}\left(\mathcal{D}_{\alpha}^{p} \cap \mathcal{B}\right)=\mathcal{C}_{\mathcal{B}}\left(\mathcal{D}_{\alpha}^{p}\right) \subset \mathcal{C}_{\mathcal{B}}\left(B^{p}\right)=\mathcal{B}_{0}
$$

Now it is clear that the polynomials lie in $\mathcal{D}_{\alpha}^{p}$ and then it follows that $\mathcal{B}_{0} \subset \mathcal{C}_{\mathcal{B}}\left(\mathcal{D}_{\alpha}^{p}\right)$. Consequently, we have

$$
\mathcal{C}_{\mathcal{B}}\left(\mathcal{D}_{\alpha}^{p} \cap \mathcal{B}\right)=\mathcal{B}_{0}, \quad 0<p<\infty, \quad \alpha \leq p-2 .
$$

If remains to consider the case where $p-2<\alpha \leq p-1$ and we shall pay a special attention to the case $\alpha=p-1$ because the spaces $\mathcal{D}_{p-1}^{p}$ are the closest ones to Hardy spaces among all the $\mathcal{D}_{\alpha}^{p}$-spaces. By the Littlewood-Paley identity, we have $\mathcal{D}_{1}^{2}=H^{2}$. We have also [21]

$$
H^{p} \subsetneq \mathcal{D}_{p-1}^{p}, \quad \text { for } 2<p<\infty
$$

and $[11,27]$

$$
\mathcal{D}_{p-1}^{p} \subsetneq H^{p}, \quad \text { for } 0<p<2 \text {. }
$$

A number of similarities and differences between the spaces $H^{p}$ and $\mathcal{D}_{p-1}^{p}$ are presented in $[4,16,17,23,27]$. As in the case of Hardy spaces, there is no inclusion relation between the spaces $\mathcal{D}_{p-1}^{p}$ and the Bloch space. Despite the fact that there 
is no relation of inclusion between $\mathcal{D}_{p-1}^{p}$ and $\mathcal{D}_{q-1}^{q}$ for $p \neq q$ (see $[4,17,12]$ ), it was observed in [7] that

$$
\mathcal{D}_{p-1}^{p} \cap \mathcal{B} \subset \mathcal{D}_{q-1}^{q} \cap \mathcal{B}, \quad 0<p<q<\infty .
$$

In the next theorem we give a characterization of the closures in the Bloch norm of the spaces $\mathcal{D}_{p-1}^{p} \cap \mathcal{B}(1 \leq p<\infty)$. We remark that, contrary to what happens with Hardy spaces, these closures are independent of $p$.

Theorem 1. Let $p \in[1, \infty)$ and $f \in \mathcal{B}$. Then the following conditions are equivalent.

(i) $f \in \mathcal{C}_{\mathcal{B}}\left(\mathcal{D}_{p-1}^{p} \cap \mathcal{B}\right)$.

(ii) For every $\varepsilon>0$

$$
\int_{\Omega_{\varepsilon}(f)} \frac{d A(z)}{1-|z|^{2}}<\infty
$$

(iii) $f \in \mathcal{C}_{\mathcal{B}}\left(H^{2} \cap \mathcal{B}\right)$.

As remarked in [22], the equivalence (ii) $\Leftrightarrow$ (iii) follows immediately from the case where $p=2$ in Theorem A by using Fubini's theorem. Indeed, using Fubini's theorem, for $f \in \mathcal{B}, \varepsilon>0$, and $a>1$, we have

$$
\begin{aligned}
& \int_{\partial \mathbf{D}} \int_{\Gamma_{a}(\xi) \cap \Omega_{\varepsilon}(f)} \frac{1}{\left(1-|z|^{2}\right)^{2}} d A(z)|d \xi| \\
& =\int_{\partial \mathbf{D}} \int_{\Omega_{\varepsilon}(f)} \chi_{\Gamma_{a}(\xi)}(z) \frac{1}{\left(1-|z|^{2}\right)^{2}} d A(z)|d \xi| \\
& =\int_{\Omega_{\varepsilon}(f)}\left(\int_{\partial \mathbf{D}} \chi_{\Gamma_{a}(\xi)}(z)|d \xi|\right) \frac{1}{\left(1-|z|^{2}\right)^{2}} d A(z) \\
& \asymp \int_{\Omega_{\varepsilon}(f)}\left(1-|z|^{2}\right) \frac{d A(z)}{\left(1-|z|^{2}\right)^{2}}=\int_{\Omega_{\varepsilon}(f)} \frac{d A(z)}{1-|z|^{2}} .
\end{aligned}
$$

Bearing in mind that (ii) $\Longleftrightarrow$ (iii), Theorem 1 follows from the following one where we give a characterization of $\mathcal{C}_{\mathcal{B}}\left(\mathcal{D}_{\alpha}^{p} \cap \mathcal{B}\right)$ whenever $1 \leq p<\infty$ and $p-2<\alpha \leq p-1$.

Theorem 2. Suppose that $1 \leq p<\infty, p-2<\alpha \leq p-1$, and let $f$ be a Bloch function. Then the following conditions are equivalent.

(i) $f \in \mathcal{C}_{\mathcal{B}}\left(\mathcal{D}_{\alpha}^{p} \cap \mathcal{B}\right)$.

(ii) For every $\varepsilon>0$ we have that

$$
\int_{\Omega_{\varepsilon}(f)} \frac{d A(z)}{\left(1-|z|^{2}\right)^{p-\alpha}}<\infty
$$

The proof of Theorem 2 will be presented in Section 2. In Section 3 we discuss the case $0<p<1$ and we study also the membership of Blaschke products in the spaces $\mathcal{C}_{\mathcal{B}}\left(\mathcal{D}_{\alpha}^{p} \cap \mathcal{B}\right)$.

We close this section noticing that, as usual, we shall be using the convention that $C=C(p, \alpha, q, \beta, \ldots)$ will denote a positive constant which depends only upon the displayed parameters $p, \alpha, q, \beta \ldots$ (which sometimes will be omitted) but not necessarily the same at different occurrences. Moreover, for two real-valued functions $E_{1}, E_{2}$ we write $E_{1} \lesssim E_{2}$, or $E_{1} \gtrsim E_{2}$, if there exists a positive constant $C$ independent of the arguments such that $E_{1} \leq C E_{2}$, respectively $E_{1} \geq C E_{2}$. If we have $E_{1} \lesssim E_{2}$ and $E_{1} \gtrsim E_{2}$ simultaneously then we say that $E_{1}$ and $E_{2}$ are equivalent and we write $E_{1} \asymp E_{2}$. 


\section{Proof of Theorem 2}

We start recalling a well known lemma (see [31, Lemma 3.10, p. 55]).

Lemma A. Suppose that $c$ is real and $t>-1$, and set

$$
F(z)=\int_{\mathbf{D}} \frac{\left(1-|w|^{2}\right)^{t}}{|1-\bar{w} z|^{2+t+c}} d A(w), \quad z \in \mathbf{D}
$$

(i) If $c<0$, then $F(z)$ is a bounded function of $z$

(ii) If $c>0$, then $F(z) \asymp\left(1-|z|^{2}\right)^{-c},|z| \rightarrow 1^{-}$.

(iii) If $c=0$, then $F(z) \asymp \log \frac{1}{\left(1-|z|^{2}\right)},|z| \rightarrow 1^{-}$.

We shall also need the following representation formula for Bloch functions (see [31, Proposition 4.27 and p. 112]).

Proposition A. Let $f$ be a Bloch function with $f(0)=f^{\prime}(0)=0$, then

$$
f(z)=\int_{\mathbf{D}} \frac{\left(1-|w|^{2}\right) f^{\prime}(w)}{(1-z \bar{w})^{2} \bar{w}} d A(w), \quad z \in \mathbf{D} .
$$

Proof of the implication (i) $\Longrightarrow$ (ii) in Theorem 2. Take a function $f$ in the closure in the Bloch norm of $\mathcal{D}_{\alpha}^{p} \cap \mathcal{B}$ and $\varepsilon>0$. Then there exists a function $g \in \mathcal{D}_{\alpha}^{p} \cap \mathcal{B}$ such that $\|f-g\|_{\mathcal{B}}<\frac{\varepsilon}{2}$. Clearly, this implies that $\Omega_{\varepsilon}(f) \subseteq \Omega_{\frac{\varepsilon}{2}}(g)$. Then it follows that

$$
\begin{aligned}
\int_{\mathbf{D}}\left|g^{\prime}(z)\right|^{p}\left(1-|z|^{2}\right)^{\alpha} d A(z) & \geq \int_{\Omega_{\frac{\varepsilon}{2}}(g)}\left|g^{\prime}(z)\right|^{p}\left(1-|z|^{2}\right)^{\alpha} d A(z) \\
& =\int_{\Omega_{\frac{\varepsilon}{2}}(g)} \frac{\left|g^{\prime}(z)\right|^{p}\left(1-|z|^{2}\right)^{p}}{\left(1-|z|^{2}\right)^{p-\alpha}} d A(z) \\
& \geq\left(\frac{\varepsilon}{2}\right)^{p} \int_{\Omega_{\frac{\varepsilon}{2}(g)}} \frac{d A(z)}{\left(1-|z|^{2}\right)^{p-\alpha}} \\
& \geq\left(\frac{\varepsilon}{2}\right)^{p} \int_{\Omega_{\varepsilon}(f)} \frac{d A(z)}{\left(1-|z|^{2}\right)^{p-\alpha}}
\end{aligned}
$$

Since $g \in \mathcal{D}_{\alpha}^{p}$, (ii) follows.

Proof of the implication (ii) $\Longrightarrow$ (i) in Theorem 2. Suppose that $1 \leq p<\infty$, $p-2<\alpha \leq p-1$, and let $f$ be a Bloch function which satisfies (ii). Assume without loss of generality that $f(0)=f^{\prime}(0)=0$. Using Proposition A we can write $f$ as follows

$$
f(z)=\int_{\mathbf{D}} \frac{\left(1-|w|^{2}\right) f^{\prime}(w)}{(1-z \bar{w})^{2} \bar{w}} d A(w), \quad z \in \mathbf{D} .
$$

Take $\varepsilon>0$. We decompose $f$ in the following way

$$
\begin{aligned}
f(z) & =\int_{\Omega_{\varepsilon}(f)} \frac{\left(1-|w|^{2}\right) f^{\prime}(w)}{(1-\bar{w} z)^{2} \bar{w}} d A(w)+\int_{\mathbf{D} \backslash \Omega_{\varepsilon}(f)} \frac{\left(1-|w|^{2}\right) f^{\prime}(w)}{(1-\bar{w} z)^{2} \bar{w}} d A(w) \\
& =f_{1}(z)+f_{2}(z) .
\end{aligned}
$$


For any $z \in \mathbf{D}$, we have

$$
\begin{aligned}
\left(1-|z|^{2}\right)\left|f_{2}^{\prime}(z)\right| & \leq 2\left(1-|z|^{2}\right) \int_{\mathbf{D} \backslash \Omega_{\varepsilon}(f)} \frac{\left(1-|w|^{2}\right)\left|f^{\prime}(w)\right|}{|1-\bar{w} z|^{3}} d A(w) \\
& \leq 2 \varepsilon\left(1-|z|^{2}\right) \int_{\mathbf{D} \backslash \Omega_{\varepsilon}(f)} \frac{d A(w)}{|1-\bar{w} z|^{3}} \\
& \leq 2 \varepsilon\left(1-|z|^{2}\right) \int_{\mathbf{D}} \frac{d A(w)}{|1-\bar{w} z|^{3}} .
\end{aligned}
$$

Using Lemma A with $t=0$ and $c=1$, we obtain that $\left(1-|z|^{2}\right)\left|f_{2}^{\prime}(z)\right| \leq C \varepsilon$ where $C$ is a positive constant. Hence, $\left\|f_{2}\right\|_{\mathcal{B}} \leq C \varepsilon$. Equivalently, $f_{1}$ is a Bloch function with

$$
\left\|f-f_{1}\right\|_{\mathcal{B}} \leq C \varepsilon \text {. }
$$

The proof will be finished if we prove that $f_{1} \in \mathcal{D}_{\alpha}^{p}$ or, equivalently, that $f_{1}^{\prime} \in A_{\alpha}^{p}$. We have

$$
\begin{aligned}
& \int_{\mathbf{D}}\left(1-|z|^{2}\right)^{\alpha}\left|f_{1}^{\prime}(z)\right|^{p} d A(z)=\int_{\mathbf{D}}\left(1-|z|^{2}\right)^{\alpha}\left|f_{1}^{\prime}(z)\right|^{p-1}\left|f_{1}^{\prime}(z)\right| d A(z) \\
& =\int_{\mathbf{D}}\left(1-|z|^{2}\right)^{p-1}\left|f_{1}^{\prime}(z)\right|^{p-1}\left(1-|z|^{2}\right)^{\alpha-p+1}\left|f_{1}^{\prime}(z)\right| d A(z) \\
& \leq\left\|f_{1}\right\|_{\mathcal{B}}^{p-1} \int_{\mathbf{D}}\left(1-|z|^{2}\right)^{\alpha-p+1}\left|f_{1}^{\prime}(z)\right| d A(z) \\
& \leq\left\|f_{1}\right\|_{\mathcal{B}}^{p-1} \int_{\mathbf{D}}\left(1-|z|^{2}\right)^{\alpha-p+1}\left(\int_{\Omega_{\varepsilon}(f)} \frac{\left(1-|w|^{2}\right)\left|f^{\prime}(w)\right|}{|1-\bar{w} z|^{3}} d A(w)\right) d A(z) \\
& \leq\left\|f_{1}\right\|_{\mathcal{B}}^{p-1}\|f\|_{\mathcal{B}} \int_{\Omega_{\varepsilon}(f)}\left(\int_{\mathbf{D}} \frac{\left(1-|z|^{2}\right)^{\alpha-p+1}}{|1-\bar{w} z|^{3}} d A(z)\right) d A(w) .
\end{aligned}
$$

Observe that $\alpha-p+1>-1$ and $p-\alpha>0$. Then, using Lemma A with $t=\alpha-p+1$ and $c=p-\alpha$ and (ii), we obtain

$$
\int_{\mathbf{D}}\left(1-|z|^{2}\right)^{\alpha}\left|f_{1}^{\prime}(z)\right|^{p} d A(z) \lesssim\left\|f_{1}\right\|_{\mathcal{B}}^{p-1}\|f\|_{\mathcal{B}} \int_{\Omega_{\varepsilon}(f)} \frac{d A(z)}{\left(1-|z|^{2}\right)^{p-\alpha}}<\infty
$$

that is, $f_{1}^{\prime} \in A_{\alpha}^{p}$ as desired.

\section{The case $0<p<1$ and some further results}

Putting together (1.3), (1.4) and Theorem 2 we have the following result.

Theorem 3. Suppose that $0<p<\infty$ and $\alpha>-1$.

(i) If $\alpha \leq p-2$, then $\mathcal{C}_{\mathcal{B}}\left(\mathcal{D}_{\alpha}^{p} \cap \mathcal{B}\right)=\mathcal{C}_{\mathcal{B}}\left(\mathcal{D}_{\alpha}^{p}\right)=\mathcal{B}_{0}$.

(ii) If $\alpha>p-1$, then $\mathcal{C}_{\mathcal{B}}\left(\mathcal{D}_{\alpha}^{p} \cap \mathcal{B}\right)=\mathcal{B}$.

(iii) If $p \geq 1$ and $p-2<\alpha \leq p-1$, then

$$
\mathcal{C}_{\mathcal{B}}\left(\mathcal{D}_{\alpha}^{p} \cap \mathcal{B}\right)=\left\{f \in \mathcal{B}: \int_{\Omega_{\varepsilon}(f)} \frac{d A(z)}{\left(1-|z|^{2}\right)^{p-\alpha}}<\infty \text { for all } \varepsilon>0\right\} .
$$

We do not know whether (iii) remains true for $0<p<1$. In particular, we do not know whether $\mathcal{C}_{\mathcal{B}}\left(\mathcal{D}_{p-1}^{p} \cap \mathcal{B}\right)$ coincides with $\mathcal{C}_{\mathcal{B}}\left(H^{2} \cap \mathcal{B}\right)$ when $0<p<1$.

We can prove the following result.

Theorem 4. Suppose that $0<p<1,-1<\alpha \leq p-1$, and let $f$ be a Bloch function.

(a) If $f \in \mathcal{C}_{\mathcal{B}}\left(\mathcal{D}_{\alpha}^{p} \cap \mathcal{B}\right)$, then $\int_{\Omega_{\varepsilon}(f)} \frac{d A(z)}{\left(1-|z|^{2}\right)^{p-\alpha}}<\infty$ for every $\varepsilon>0$. 
(b) If there exists $\gamma>2-\frac{1+\alpha}{p}$ such that $\int_{\Omega_{\varepsilon}(f)} \frac{d A(z)}{\left(1-|z|^{2}\right)^{\gamma}}<\infty$ for every $\varepsilon>0$, then $f \in \mathcal{C}_{\mathcal{B}}\left(\mathcal{D}_{\alpha}^{p} \cap \mathcal{B}\right)$.

Proof. For $f \in \mathcal{B}$, we have

$$
\begin{aligned}
\int_{\mathbf{D}}\left(1-|z|^{2}\right)^{\alpha+1-p}\left|f^{\prime}(z)\right| d A(z) & =\int_{\mathbf{D}}\left(1-|z|^{2}\right)^{\alpha}\left|f^{\prime}(z)\right|^{p}\left[\left(1-|z|^{2}\right)\left|f^{\prime}(z)\right|\right]^{1-p} d A(z) \\
& \leq\|f\|_{\mathcal{B}}^{1-p} \int_{\mathbf{D}}\left(1-|z|^{2}\right)^{\alpha}\left|f^{\prime}(z)\right|^{p} d A(z) .
\end{aligned}
$$

Hence, it follows that $\mathcal{D}_{\alpha}^{p} \cap \mathcal{B} \subset \mathcal{D}_{\alpha+1-p}^{1} \cap \mathcal{B}$. Using this, the fact that $-1<\alpha+1-p \leq$ 0 , and Theorem 2, (a) follows.

We turn to prove (b). Observe that

$$
1 \leq 2-\frac{1+\alpha}{p}<2
$$

Suppose that $\gamma>2-\frac{1+\alpha}{p}$ and that $\int_{\Omega_{\varepsilon}(f)} \frac{d A(z)}{\left(1-|z|^{2}\right)^{\gamma}}<\infty$ for every $\varepsilon>0$. Clearly, we may assume without loss of generality that $\gamma<2$. Arguing as is the proof of the implication (ii) $\Longrightarrow$ (i) in Theorem 2, the fact $f \in \mathcal{C}_{\mathcal{B}}\left(\mathcal{D}_{p-1}^{p} \cap \mathcal{B}\right)$ will follow if we prove that the Bloch function $f_{1}$ defined by

$$
f_{1}(z)=\int_{\Omega_{\varepsilon}(f)} \frac{\left(1-|w|^{2}\right) f^{\prime}(w)}{(1-\bar{w} z)^{2} \bar{w}} d A(w), \quad z \in \mathbf{D},
$$

belongs to the space $\mathcal{D}_{\alpha}^{p}$ or, equivalently, that

$$
f_{1}^{\prime} \in A_{\alpha}^{p} .
$$

We are going to present two proofs of (3.1), the second one has been suggested to us by one of the referees. Observe that $0<2-\gamma<\frac{\alpha+1}{p}$ and $1-\gamma>-1$. Then it follows that $A_{1-\gamma}^{1} \subset A_{\alpha}^{p}$ (see [20, p. 703] or [6, Lemma 1.2])). Hence it suffices to show that

$$
f_{1}^{\prime} \in A_{1-\gamma}^{1} \text {. }
$$

We have

$$
\begin{aligned}
& \int_{\mathbf{D}}\left(1-|z|^{2}\right)^{1-\gamma}\left|f_{1}^{\prime}(z)\right| d A(z) \\
& \leq \int_{\mathbf{D}}\left(1-|z|^{2}\right)^{1-\gamma} \int_{\Omega_{\varepsilon}(f)} \frac{\left(1-|w|^{2}\right)\left|f^{\prime}(w)\right|}{|1-\bar{w} z|^{3}} d A(w) d A(z) \\
& \leq\|f\|_{\mathcal{B}} \int_{\Omega_{\varepsilon}(f)}\left(\int_{\mathbf{D}} \frac{\left(1-|z|^{2}\right)^{1-\gamma}}{|1-\bar{w} z|^{3}} d A(z)\right) d A(w) \\
& \leq\|f\|_{\mathcal{B}} \int_{\Omega_{\varepsilon}(f)} \frac{d A(w)}{\left(1-|w|^{2}\right)^{\gamma}} .
\end{aligned}
$$

To obtain the last inequality we have used Lemma A with $t=1-\gamma$ and $c=\gamma$. Then (3.2) follows.

Let us turn to the other promised proof of (3.1). Notice that

$$
0<(1-p)(\alpha+1)<p(1-\alpha) \text {. }
$$

Pick $\delta$ with

$$
0<\delta<(1-p)(\alpha+1)
$$


and define $h(z)=\left(1-|z|^{2}\right)^{\delta}(z \in \mathbf{D})$. Using Hölder's inequality, Fubini's theorem, the facts that $\frac{\delta}{1-p}-\alpha<1, \alpha+\frac{\delta}{p}>-1$ and $1-\alpha-\frac{\delta}{p}>0$, and Lemma A, we obtain

$$
\begin{aligned}
& \int_{\mathbf{D}}\left|f_{1}^{\prime}(z)\right|^{p}\left(1-|z|^{2}\right)^{\alpha} d A(z) \lesssim \int_{\mathbf{D}}\left(\int_{\Omega_{\varepsilon}(f)} \frac{\left|f^{\prime}(w)\right|\left(1-|w|^{2}\right)}{|1-\bar{w} z|^{3}} d A(w)\right)^{p}\left(1-|z|^{2}\right)^{\alpha} d A(z) \\
& \leq\|f\|_{\mathcal{B}}^{p} \int_{\mathbf{D}}\left(\int_{\Omega_{\varepsilon}(f)} \frac{d A(w)}{|1-\bar{w} z|^{3}}\right)^{p} h(z) h(z)^{-1}\left(1-|z|^{2}\right)^{\alpha p}\left(1-|z|^{2}\right)^{\alpha(1-p)} d A(z) \\
& \lesssim\|f\|_{\mathcal{B}}^{p}\left(\int_{\mathbf{D}}\left(1-|z|^{2}\right)^{\alpha+\frac{\delta}{p}} \int_{\Omega_{\varepsilon}(f)} \frac{d A(w)}{|1-\bar{w} z|^{3}} d A(z)\right)^{p}\left(\int_{\mathbf{D}}\left(1-|z|^{2}\right)^{\alpha-\frac{\delta}{1-p}} d A(z)\right)^{1-p} \\
& \lesssim\|f\|_{\mathcal{B}}^{p}\left(\int_{\Omega_{\varepsilon}(f)} \int_{\mathbf{D}} \frac{\left(1-|z|^{2}\right)^{\alpha+\frac{\delta}{p}}}{|1-\bar{w} z|^{3}} d A(z) d A(w)\right)^{p} \lesssim\|f\|_{\mathcal{B}}^{p}\left(\int_{\Omega_{\varepsilon}(f)} \frac{d A(w)}{\left(1-|w|^{2}\right)^{1-\alpha-\frac{\delta}{p}}}\right)^{p} .
\end{aligned}
$$

Since $1-\alpha-\frac{(1-p)(\alpha+1)}{p}=2-\frac{\alpha+1}{p}$, (3.1) follows choosing $\delta$ sufficiently close to $(1-p)(\alpha+1)$.

Our next aim is to give applications of the results that we have obtained so far to study the membership of a Blaschke product in $\mathcal{C}_{\mathcal{B}}\left(\mathcal{D}_{\alpha}^{p} \cap \mathcal{B}\right)$ for distinct values of $p$ and $\alpha$. We refer to [9] for the definition, notation, and results about Blaschke products. Since $H^{\infty} \subset H^{2} \cap \mathcal{B}$, Theorem 1 trivially implies that

$$
H^{\infty} \subset \mathcal{C}_{\mathcal{B}}\left(\mathcal{D}_{p-1}^{p} \cap \mathcal{B}\right), \quad 1 \leq p<\infty .
$$

In particular any Blaschke product lies in $\mathcal{C}_{\mathcal{B}}\left(\mathcal{D}_{p-1}^{p} \cap \mathcal{B}\right)$ whenever $1 \leq p<\infty$.

For $0<p<2$ the space $H^{\infty}$ is not included in $\mathcal{D}_{p-1}^{p}$. Rudin [25, Theorem III] proved that there exists a Blaschke product $B$ with $B \notin \mathcal{D}_{0}^{1}$. Later on, Vinogradov [27] gave examples of Blaschke products $B$ such that $B \notin \mathcal{D}_{p-1}^{p}$ for all $p \in(0,2)$.

On the other hand, Rudin also proved in [25] that if a sequence $\left\{a_{n}\right\} \subset \mathbf{D}$ satisfies the condition

$$
\sum\left(1-\left|a_{n}\right|\right) \log \frac{1}{1-\left|a_{n}\right|}<\infty
$$

then the Blaschke product whose sequence of zeros is $\left\{a_{n}\right\}$ belongs to $\mathcal{D}_{0}^{1}$ (and, consequently to $\mathcal{D}_{p-1}^{p}$ for all $p \geq 1$ ). The converse of this is not true. Indeed, a result of Vinogradov [27, Theorem 2.9, p. 3814] implies that a Blaschke product with zeros in a Stolz angle lies in $\mathcal{D}_{p-1}^{p}$ for all $p$.

Protas proved in [24, Theorem 1] that if $0<s<1$ and the sequence $\left\{a_{n}\right\}$ of the zeros of the Blaschke product $B$ satisfies the condition $\sum\left(1-\left|a_{n}\right|^{2}\right)^{s}<\infty$, then $B^{\prime} \in A_{s-1}^{1}$. Using again [6, Lemma 1.2] we see that $A_{s-1}^{1} \subset A_{p-1}^{p}$ for all $p \in(0,1)$, whenever $0<s<1$. Then we deduce the following:

If the sequence $\left\{a_{n}\right\}$ of the zeros of the Blaschke product $B$ satisfies the condition $\sum\left(1-\left|a_{n}\right|^{2}\right)^{s}<\infty$ for some $s<1$, then $B \in \cap_{0<p<\infty} \mathcal{D}_{p-1}^{p}$.

Let us summarize these facts in the following theorem.

Theorem 5. Let $B$ be a Blaschke product and let $\left\{a_{n}\right\}$ be its sequence of zeros.

(i) $B \in \mathcal{C}_{\mathcal{B}}\left(\mathcal{D}_{p-1}^{p} \cap \mathcal{B}\right)$ whenever $1 \leq p<\infty$.

(ii) If $\sum\left(1-\left|a_{n}\right|\right) \log \frac{1}{1-\left|a_{n}\right|}<\infty$, then $B \in \cap_{1 \leq p<\infty} \mathcal{D}_{p-1}^{p}$.

(iii) If $\sum\left(1-\left|a_{n}\right|^{2}\right)^{s}<\infty$ for some $s<1$, then $B \in \cap_{0<p<\infty} \mathcal{D}_{p-1}^{p}$. 
Suppose that $1 \leq \gamma<2$ and let $B$ be the Blaschke product whose sequence of zeros is $\left\{a_{n}\right\}$. Take $\varepsilon>0$. We have

$$
\left|B^{\prime}(z)\right| \leq \sum \frac{1-\left|a_{n}\right|^{2}}{\left|1-\overline{a_{n}} z\right|^{2}}, \quad z \in \mathbf{D},
$$

and hence

$$
z \in \Omega_{\varepsilon}(B) \quad \Longrightarrow \quad 1 \leq \frac{1}{\varepsilon}\left(1-|z|^{2}\right) \sum \frac{1-\left|a_{n}\right|^{2}}{\left|1-\overline{a_{n}} z\right|^{2}} .
$$

Then it follows that

$$
\begin{aligned}
\int_{\Omega_{\varepsilon}(B)} \frac{d A(z)}{\left(1-|z|^{2}\right)^{\gamma}} & \leq \frac{1}{\varepsilon} \sum\left(1-\left|a_{n}\right|^{2}\right) \int_{\Omega_{\varepsilon}(B)} \frac{\left(1-|z|^{2}\right)^{1-\gamma}}{\left|1-\overline{a_{n}} z\right|^{2}} d A(z) \\
& \leq \frac{1}{\varepsilon} \sum\left(1-\left|a_{n}\right|^{2}\right) \int_{\mathbf{D}} \frac{\left(1-|z|^{2}\right)^{1-\gamma}}{\left|1-\overline{a_{n}} z\right|^{2}} d A(z) .
\end{aligned}
$$

Now, using Lemma A with $t=1-\gamma$ and $c=\gamma-1$, we obtain

$$
\int_{\Omega_{\varepsilon}(B)} \frac{d A(z)}{1-|z|^{2}} \lesssim \frac{1}{\varepsilon} \sum\left(1-\left|a_{n}\right|^{2}\right) \log \frac{1}{1-\left|a_{n}\right|^{2}}
$$

and

$$
\int_{\Omega_{\varepsilon}(B)} \frac{d A(z)}{\left(1-|z|^{2}\right)^{\gamma}} \lesssim \frac{1}{\varepsilon} \sum\left(1-\left|a_{n}\right|^{2}\right)^{2-\gamma}, \quad \text { if } 1<\gamma<2 .
$$

Using these inequalities and Theorem 1 and Theorem 4 with $\alpha=p-1$, we obtain results which are weaker than those stated in Theorem 5. However, using (3.7) and Theorem 4 in the case $\alpha<p-1$, we obtain the following result.

Theorem 6. Let $B$ be the Blaschke product whose sequence of zeros is $\left\{a_{n}\right\}$. If $1 \leq p<\infty, p-2<\alpha<p-1$, and $\sum\left(1-\left|a_{n}\right|^{2}\right)^{2-(p-\alpha)}<\infty$, then $B \in \mathcal{C}_{\mathcal{B}}\left(\mathcal{D}_{\alpha}^{p} \cap \mathcal{B}\right)$.

Restricting ourselves to interpolating Blaschke products (that is, Blaschke products whose sequences of zeros are universal interpolation sequences [9, Chapter 9]), we have the following result.

Theorem 7. Let $B$ be an interpolating Blaschke product whose sequence of zeros is $\left\{a_{n}\right\}_{n=1}^{\infty}$. Suppose that $1 \leq p<\infty$ and $p-2<\alpha<p-1$. Then the following conditions are equivalent.

(i) $\sum\left(1-\left|a_{n}\right|^{2}\right)^{2-(p-\alpha)}<\infty$.

(ii) $B \in \mathcal{C}_{\mathcal{B}}\left(\mathcal{D}_{\alpha}^{p} \cap \mathcal{B}\right)$.

We remark that this was proved in [5] for the case where $p=2$ and $0<\alpha<1$.

Proof of Theorem 7. The implication (i) $\Longrightarrow$ (ii) follows trivially from Theorem 6 . To prove the other implication, suppose that $B \in \mathcal{C}_{\mathcal{B}}\left(\mathcal{D}_{\alpha}^{p} \cap \mathcal{B}\right)$. By Theorem 3 we have

$$
\int_{\Omega_{\varepsilon}(f)} \frac{d A(z)}{\left(1-|z|^{2}\right)^{p-\alpha}}<\infty .
$$

Since $B$ is an interpolating Blaschke product, the sequence $\left\{a_{n}\right\}$ is uniformly separated, that is, there exists $\delta>0$ such that

$$
\inf _{m \geq 1} \prod_{n=1, n \neq m}^{\infty} \varrho\left(a_{n}, a_{m}\right) \geq \delta .
$$


Here $\varrho$ denotes the pseudo-hyperbolic distance:

$$
\varrho(z, w)=\left|\frac{z-w}{1-\bar{w} z}\right|, \quad z, w \in \mathbf{D} .
$$

Also, for $a \in \mathbf{D}$ and $0<r<1, \Delta(a, r)$ will denote the pseudo-hyperbolic disc of center $a$ and radius $r$ :

$$
\Delta(a, r)=\{z \in \mathbf{D}: \varrho(z, a)<r\} .
$$

Using Lemma 3.5 of [18] we see that there exist $\varepsilon>0$ and $\beta \in(0,1)$ such that the $\operatorname{discs}\left\{\Delta\left(a_{n}, \beta\right): n=1,2,3, \ldots\right\}$ are pairwise disjoint and so that

$$
\left|B^{\prime}(z)\right| \geq \frac{\varepsilon}{1-\left|a_{n}\right|^{2}}, \quad z \in \Delta\left(a_{n}, \beta\right), \quad n=1,2,3, \ldots
$$

This implies that

$$
\bigcup_{n=1}^{\infty} \Delta\left(a_{n}, \beta\right) \subset \Omega_{\varepsilon}(B) .
$$

Using the fact that the discs $\left\{\Delta\left(a_{n}, \beta\right)\right\}$ are pairwise disjoint and (3.9), we obtain

$$
\sum_{n=1}^{\infty} \int_{\Delta\left(a_{n}, \beta\right)} \frac{d A(z)}{\left(1-|z|^{2}\right)^{p-\alpha}}=\int_{\cup_{n=1}^{\infty} \Delta\left(a_{n}, \beta\right)} \frac{d A(z)}{\left(1-|z|^{2}\right)^{p-\alpha}} \leq \int_{\Omega_{\varepsilon}(B)} \frac{d A(z)}{\left(1-|z|^{2}\right)^{p-\alpha}}
$$

Now, (see [31, p. 69]) it is well known that

$$
\left(1-|z|^{2}\right) \asymp\left(1-\left|a_{n}\right|^{2}\right), \quad \text { as long as } z \in \Delta\left(a_{n}, \beta\right),
$$

and that the area $A\left(\Delta\left(a_{n}, \beta\right)\right)$ of $\Delta\left(a_{n}, \beta\right)$ satisfies $A\left(\Delta\left(a_{n}, \beta\right)\right) \asymp\left(1-\left|a_{n}\right|^{2}\right)^{2}$. These two facts imply that

$$
\sum_{n=1}^{\infty}\left(1-\left|a_{n}\right|^{2}\right)^{2-(p-\alpha)} \asymp \sum_{n=1}^{\infty} \int_{\Delta\left(a_{n}, \beta\right)} \frac{d A(z)}{\left(1-|z|^{2}\right)^{p-\alpha}} .
$$

This, together with (3.10) and (3.8), implies that $\sum_{n=1}^{\infty}\left(1-\left|a_{n}\right|^{2}\right)^{2-(p-\alpha)}<\infty$.

Acknowledgements. We wish to express our gratitude to the referees for their helpful comments and suggestions which have lead us to improve the paper.

\section{References}

[1] Anderson, J. M., J. Clunie, and Ch. Pommerenke: On Bloch functions and normal functions. - J. Reine Angew. Math. 270, 1974, 12-37.

[2] Anderson, J. M.: Bloch functions: the basic theory. - In: Operators and function theory (Lancaster, 1984), NATO Adv. Sci. Inst. Ser. C Math. Phys. Sci. 153, Reidel, Dordrecht, 1985, $1-17$.

[3] Arazy, J., S. D. Fisher, and J. Peetre: Möbius invariant function spaces. - J. Reine Angew. Math. 363, 1985, 110-145.

[4] Baernstein, A., D. Girela, and J.A. Peláez: Univalent functions, Hardy spaces and spaces of Dirichlet type. - Illinois J. Math. 48:3, 2004, 837-859.

[5] BAO, G., and N. G. GöĞÜş: On the closures of Dirichlet type spaces in the Bloch space. Complex Anal. Oper. Theory (to appear).

[6] Buckley, S. M., P. Koskela, and D. Vukotić: Fractional integration, differentiation, and weighted Bergman spaces. - Math. Proc. Cambridge Philos. Soc. 126:2, 1999, 369-385.

[7] Chatzifountas, Ch., D. Girela, and J. A. Peláez: Multipliers of Dirichlet subspaces of the Bloch space. - J. Operator Theory 72:1, 2014, 159-191. 
[8] Donaire, J. J., D. Girela, and D. Vukotić: On univalent functions in some Möbius invariant spaces. - J. Reine Angew. Math. 553, 2002, 43-72.

[9] Duren, P.Ł.: Theory of $H^{p}$ spaces. - Academic Press, New York-London, 1970.

[10] Duren, P. L., and A. P. Schuster: Bergman spaces. - Math. Surveys Monogr. 100, Amer. Math. Soc., Providence, Rhode Island, 2004.

[11] Flett, T. M.: The dual of an inequality of Hardy and Littlewood and some related inequalities. - J. Math. Anal. Appl. 38, 1972, 746-765.

[12] Galanopoulos, P., D. Girela, and J. A. Peláez: Multipliers and integration operators on Dirichlet spaces. - Trans. Amer. Math. Soc. 363:4, 2011, 1855-1886.

[13] Galanopoulos, P., N. Monreal Galán, and J. Pau: Closure of Hardy spaces in the Bloch space. - J. Math. Anal. Appl. 429:2, 2015, 1214-1221.

[14] Ghatage, P. G., and D. Zheng: Analytic functions of bounded mean oscillation and the Bloch space. - Integral Equations Operator Theory 17:4, 1993, 501-515.

[15] Girela, D.: Analytic functions of bounded mean oscillation. - In: Complex Function Spaces, Mekrijärvi 1999, editor R. Aulaskari, Univ. Joensuu Dept. Math. Rep. Ser. 4, Univ. Joensuu, Joensuu, 2001, 61-170.

[16] Girela, D., and J.A. Peláez: Carleson measures for spaces of Dirichlet type. - Integral Equations Operator Theory 55:3, 2006, 415-427.

[17] Girela, D., and J. A. Peláez: Growth properties and sequences of zeros of analytic functions in spaces of Dirichlet type. - J. Australian Math. Soc. 80, 2006, 397-418.

[18] Girela, D., J. A. Peláez, and D. Vukotić: Integrability of the derivative of a Blaschke product. - Proc. Edinb. Math. Soc. (2) 50:3, 2007, 673-687.

[19] Hedenmalm, H., B. Korenblum, and K. Zhu: Theory of Bergman spaces. - Grad. Texts in Math. 199, Springer, New York, Berlin, 2000.

[20] Honowitz, C.: Zeros of functions in the Bergman spaces. - Duke Math. J. 41, 1974, 693-710.

[21] Littlewood, J. E., and R. E. A. C. Paley: Theorems on Fourier series and power series (II). - Proc. London Math. Soc. 42, 1936, 52-89.

[22] Monreal Galán, N., and A. Nicolau: The closure of the Hardy space in the Bloch norm. - Algebra i Analiz 22:1, 2010, 75-81; transl. in St. Petersburg Math. J. 22:1, 2011, 55-59.

[23] Peláez, J. A., F. PÉrez-GonzÁlez, and J. RÄttyä: Operator theoretic differences between Hardy and Dirichlet-type spaces. - J. Math. Anal. Appl. 418:1, 2014, 387-402.

[24] Protas, D.: Blaschke products with derivative in $H^{p}$ and $B^{p}$. - Michigan Math. J. 20, 1973, 393-396.

[25] Rudin, W.: The radial variation of analytic functions. - Duke Math. J. 22, 1955, 235-242.

[26] TJani, M.: Distance of a Bloch function to the little Bloch space. - Bull. Austral. Math. Soc. 74:1, 2006, 101-119.

[27] Vinogradov, S. A.: Multiplication and division in the space of analytic functions with area integrable derivative, and in some related spaces. - Zap. Nauchn. Sem. S.-Peterburg. Otdel. Mat. Inst. Steklov. (POMI) 222, 1995, Issled. po Linein. Oper. i Teor. Funktsii 23, 45-77, 308 (in Russian); transl. in J. Math. Sci. (New York) 87:5, 1997, 3806-3827.

[28] WU, Z.: Carleson measures and multipliers for Dirichlet spaces. - J. Funct. Anal. 169, 1999, $148-163$.

[29] Zhao, R.: Distances from Bloch functions to some Möbius invariant spaces. - Ann. Acad. Sci. Fenn. Math. 33:1, 2008, 303-313.

[30] Zhu, K.: Analytic Besov spaces. - J. Math. Anal. Appl. 157, 1991, 318-336.

[31] ZHU, K.: Operator theory in function spaces. Second edition. - Math. Surveys Monogr. 138, Amer. Math. Soc., Providence, Rhode Island, 2007. 